\title{
Ansiedad en maratonianos en función de variables socio-demográficas Anxiety in marathoners based on socio-demographic variables
}

\author{
Francisco Ruiz-Juan* y Antonio Zarauz Sancho** \\ *Universitad de Murcia, **I.E.S.O. Azcona-Almería
}

\begin{abstract}
Resumen: En la creciente población de corredores españoles y mexicanos de ruta se analizan su ansiedad precompetitiva y sus principales características bio-socio-demográficas. También se analiza la influencia de dichas variables en la puntuación de la ansiedad cognitiva, la ansiedad somática y la autoconfianza, tanto en la muestra española de corredores de ruta, como en la mexicana. Se obtienen valiosos datos descriptivos de su ansiedad y características bio-socio-demográficas y se concluye que los corredores de ruta tienen una moderada ansiedad precompetitiva, tanto cognitiva como somática, sin diferencias significativas entre sexos ni entre las muestras española y mexicana. Sin embargo, los valores obtenidos en autoconfianza son altos en la muestra española y muy altos en la muestra mexicana, en ambos casos con diferencias significativas a favor de los varones. Por último, en la influencia que tienen las variables bio-socio-demográficas en las puntuaciones de la ansiedad cognitiva, ansiedad somática y autoconfianza, existen numerosas diferencias significativas, tanto por sexo como entre las muestras española y mexicana.
\end{abstract}

Palabras clave: CSAI-2R; variables socio-demográficas; ansiedad cognitiva; ansiedad somática; autoconfianza.

Abstract: In the growing population of Spanish and Mexican route runners we analyze their precompetitive anxiety and its main features bio-sociodemographic characteristics. We also analyze the influence of these variables on the cognitive anxiety score, somatic anxiety and self-confidence, both in the Spanish sample route corridors, as in Mexican sample. Valuable descriptive data were obtained of their anxiety and bio-socio-demographic characteristic, and concludes that runners have a moderate route precompetitive anxiety, cognitive or somatic, without significant differences between sexes or between Spanish and Mexican samples. However, the score obtained in confidence are high in the Spanish sample and very high in the Mexican sample, in both cases with significant differences in favor of males. Finally, the influence of bio-socio-demographic variables scores in cognitive anxiety, somatic anxiety and self-confidence, there are many significant differences, both by gender and between Spanish and Mexican samples.

Key words: CSAI-2R; socio-demographic variables; cognitive anxiety; somatic anxiety; self confidence.

\section{Introducción}

Anshel et al. (1991) definieron la ansiedad como un sentimiento subjetivo de aprensión o amenaza percibida, a veces acompañada por un incremento de la activación fisiológica. Jaenes (2000), en función de la estabilidad de la ansiedad, distinguía entre ansiedad rasgo (ser) como la tendencia personal a tener ansiedad en la mayoría de situaciones de la vida cotidiana, y ansiedad estado (estar) como la manifestada por una persona en una situación concreta.

Desde una aproximación multidimensional, Martens, Burton, Vealey,Bumpy Smith (1990) diferenciaron en la ansiedad precompetitiva tres componentes en función de a qué nivel se manifestaba; ansiedad cognitiva, ansiedad somática y autoconfianza. La ansiedad cognitiva evalúa las sensaciones negativas que el sujeto tiene sobre su rendimiento y las consecuencias del resultado. La ansiedad somática hace referencia a la percepción de indicadores fisiológicos de la ansiedad, como tensión muscular, aumento de frecuencia cardíaca, sudoración y malestar en el estómago. La autoconfianza estima el grado de seguridad que el sujeto cree tener acerca de sus posibilidades de éxito en la competición.

La ansiedad es un constructo psicológico ampliamente estudiado en el campo de la conducta humana en general y de la psicología del deporte en particular. El motivo son sus repercusiones posteriores en el rendimiento de los deportistas, puesto que, según controlen sus síntomas, puede aumentarles o disminuirles el rendimiento (Smith, 1989). Jones (1991) explicaba que, en función de si un deportista percibe como favorable o desfavorable los síntomas de la ansiedad precompetitiva (por ejemplo los síntomas de la ansiedad somática), puede beneficiarle o perjudicarle en su ejecución o rendimiento posterior. Así, aunque en la literatura hay trabajos en los que se concluye que la ansiedad precompetitiva facilita el rendimiento (Jones \& Swain, 1995), en la mayoría de las investigaciones se concluye que la ansiedad tiene efectos negativos no sólo en el rendimiento, si no en el disfrute de la participa-

Fecha recepción: 20-10-13- Fecha envío revisores: 29-10-13- Fecha de aceptación: 15-11-13 Francisco Ruiz Juan

C/ Argentina s/n

30720 Santiago de la Ribera-Murcia

pacoruizjuan@gmail.com ción deportiva y en el bienestar físico (Scanlan, Babkes \& Scanlan, 2005; Scanlan \& Lewthwaite, 1986; Smith, Smoll, \& Wiechman, 1998).

Existen variables de índole sociodemográfica, personal o interna que pueden influir en la ansiedad precompetitiva de un deportista. Por ejemplo, aunque los hombres muestran mayor autoconfianza que las mujeres, son ellas quienes controlan mejor su ansiedad (Ponce de León, López \& Medina, 2006). También, la menor edad(Arbinaga \& Caracuel, 2005), menor experiencia en competición (Mellalieu, Hanton \& O’Brien, 2004), la percepción del deportista de no ir adecuadamente entrenado a competir(Gutiérrez, Estévez, García \& Pérez, 1997), el menor nivel del deportista (Jones \& Swain, 1995; Pozo, 2007), o incluso la percepción de la ansiedad como desfavorecedora del rendimiento (Pozo, 2007), son sólo algunas variables que aumentan la ansiedad y disminuyen la autoconfianza.

De la misma manera, en los estados de ansiedad de los deportistas pueden influir diferentes variables deíndole externo. Fursty Tenembaum (1984), por ejemplo, encontraron que los deportistas de equipo mostraban menor ansiedad que los individuales. También, el ambiente en el que se encuentra compitiendo el deportista (Arbinaga \& Caracuel, 2005), el tipo y características de la prueba a la que se enfrenta (Jaenes, 2000), así como el tipo de deporte (Martens, et al., 1990; Pozo, 2007) son variables que pueden influir en el grado que se manifiestan los síntomas de la ansiedad.

En España, salvo por los estudios de Jaenes (2000) y Buceta, López, Pérez-Llantada, Vallejo y Del Pino (2003), no es fácil encontrar investigaciones que analicen en profundidad la ansiedad precompetitiva del creciente número de corredores de ruta. De hecho, consultando las web de los principales maratones en territorio español (Madrid y Barcelona), se puede verificar que se ha pasado de apenas unos pocos cientos de corredores que los finalizaban más de 10.000 en la actualidad. En Hispanoamérica, tampoco resulta fácil encontrar investigaciones que analicen la ansiedad en los corredores de ruta.

Por consiguiente, en la presente investigación se van a analizar una serie de variables bio-socio-demográficas y la influencia que tienen a la hora de elevar o disminuir de manera significativa la ansiedad precompetitiva en una amplia muestra de corredores de ruta hispanos (españoles y mexicanos). 


\section{Método}

\section{Participantes}

Se partió del total de corredores inscritos en los medios maratones de Almería y Elche (España) y Guadalajara (México) en 2011 y 2012. Para asegurar que la muestra fuera representativa (error $\pm 3 \%$, intervalo de confianza 95.5\%), se empleó un diseño de muestreo estratificado por afijación proporcional teniendo en cuenta el sexo (86.65\% de hombres y $13.35 \%$ de mujeres) y la edad. Se administró un cuestionario a 1054 corredores de fondo en ruta que participaron en los medios maratones de Almería (30/01/2011), Elche(03/04/2011) y Guadalajara (20/02/11; 19/02/2012).

Además, por medio de una página Web se obtuvieron las respuestas de 741 cuestionarios de corredores de maratón y medio maratón desde 01/03/11 a 20/03/13. Por tanto, la muestra aleatoria y voluntaria fue de 1795 (1105 españoles, 690 mexicanos) corredores de fondo en ruta que estaba compuesta por 1541 hombres (85.7\%) con rango de edad de 18 a 76 años $(M=38.98 ; D T=10.45)$ y 254 mujeres (14.35\%) con rango de edad de 18 a 69 años $(M=37.88 ; D T=9.80)$. Por tanto, se tiene una muestra representativa con un error del $\pm 2.30 \%$, a un intervalo de confianza del $95.5 \%$.

\section{Procedimiento}

En las carreras citadas anteriormente, se pidió permiso a la organización de la carrera mediante una carta en la que se explicaban los objetivos de la investigación, cómo se iba a realizar el estudio y se acompañaba un modelo del instrumento. El cuestionario fue administrado en un stand que se dispuso al efecto durante la recogida de dorsales de los atletas participantes el día previo a la carrera.

Para ampliar la muestra y obtener la más amplia y variada geográficamente en el territorio español, se solicitó colaboración al webmaster del principal foro de atletismo de España (http:// www.elatleta.com/foro/forum.php), al que se le pidió que colgara el cuestionario en la sección de corredores de ruta (http://www.retos.org/ encuesta/inicio.html).

En ambos casos, todos los sujetos fueron informados del objetivo del estudio, de la voluntariedad, absoluta confidencialidad de las respuestas y manejo de los datos, que no había respuestas correctas o incorrectas y se les solicitó que contestaran con la máxima sinceridad y honestidad. Además, se pidió que si habían contestado previamente este cuestionario no lo volvieran a hacer. Este trabajo posee el informe favorable de la Comisión de Bioética de la Universidad de Murcia.

\section{Instrumento}

Se administró un instrumento que estaba compuesto de:

Inventario de Ansiedad Competitiva-2 Revisado; versión española de Andrade et al. (2007) de Revised Competitive State Anxiety Inventory-2 (CSAI-2R) de Cox et al. (2003). Tiene 3 subescalas: ansiedad cognitiva, ansiedad somática y autoconfianza. La primera y tercera contienen 5 ítems puntuables de 1 (nada) a 4 (mucho) con la que se obtiene una puntuación total entre 5 y 20. La segunda contiene 6 ítems, que ofrece puntuaciones entre 6 y 24.

Variables bio-socio-demográficas; ¿tiene entrenador?, ¿Quénivel de estudios ha finalizado?, ¿con quién vive actualmente?, ¿Cuál es su actividad laboral principal?, ¿Cuántos hijos y/o familiares muy dependientes tiene a su cargo?, ¿Qué edad tiene?, ¿Cuál es su peso? y ¿Cuál es su estatura?

\section{Análisis de los datos}

Consistencia interna (alfa de Cronbach), diferencias de medias por sexo ( $t$ Student), Porcentajes, Chi-cuadrado de Pearson $\left(X^{2}\right)$, significatividad, Media (M), Desviación típica (DT), F y $p$ valor se realizaron con SPSS 20.0.

\section{Resultados}

\section{Estadística descriptiva}

Los corredores de ruta tienen una moderada ansiedad precompetitiva, tanto cognitiva como somática, sin diferencias significativas entre sexos ni entre las muestras española y mexicana. Sin embargo, los valores obtenidos en autoconfianza son altos en la muestra española y muy altos en la muestra mexicana, en ambos casos con diferencias significativas a favor de los varones (véase Tabla 1).

Con respecto a las variables bio-socio-demográficas, excepto en la edad, en la muestra mexicana hay diferencias significativas por sexo en todas las variables analizadas, mientras que en la española sólo las hay en la dedicación principal y en el IMC. Respecto a tener entrenador, una minoría de españoles se preocupa de tenerlo, mientras que en los mexicanos esta preocupación es mucho mayor, sobre todo en mujeres. La mayoría del total de la muestra tiene un alto nivel de estudios, convive con alguien, trabaja, tiene pocos o ningún hijo o familiares dependientes a su cargo, tienen un peso normal y menos de 50 años (Véase Tabla 2).

Medias en ansiedad y autoconfianza en función de las variables bio-socio-demográficas

En ansiedad cognitiva se obtuvieron valores medios en todas las opciones de respuesta de las variables analizadas, tanto en la muestra española como en la mexicana. En la muestra española, además, se obtuvieron numerosas diferencias significativas, de forma que esta ansiedad cognitiva disminuía en hombres y mujeres sin entrenador, con trabajo, con mayor sobrepeso y en el tramo de edad de 34 a 39 años (mujeres) y en mayores de 47 años (hombres). En la muestra mexicana, sin embargo, sólo hubo diferencias significativas en hombres y mujeres que no tenían entrenador, en hombres con mayor nivel de estudios y mujeres de entre 34 y 39 años, en todos los cuales su ansiedad cognitiva disminuía(Véase Tabla3).

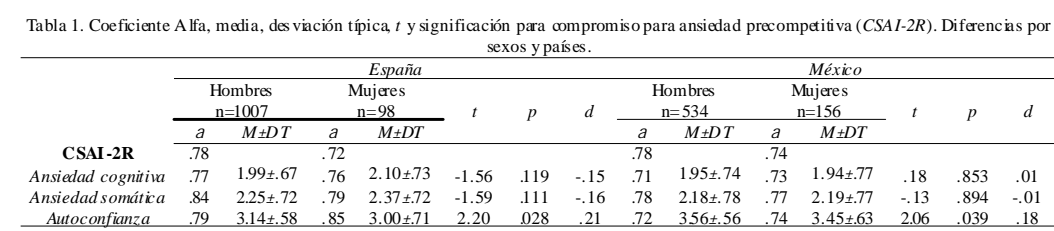

\begin{tabular}{|c|c|c|c|c|c|c|c|c|}
\hline & \multicolumn{4}{|c|}{ España } & \multicolumn{4}{|c|}{ México } \\
\hline & $\begin{array}{c}\text { Hom bres } \\
n=1007\end{array}$ & $\begin{array}{c}\text { Mujeres } \\
n=98\end{array}$ & Total & $X^{2}$ & $\begin{array}{c}\text { Hombres } \\
n=534\end{array}$ & $\begin{array}{c}\text { Mujeres } \\
n=156\end{array}$ & Total & $X^{2}$ \\
\hline \multicolumn{9}{|l|}{ Tener entrenador } \\
\hline $\mathrm{Si}$ & 15.1 & 19.4 & 15.5 & & 27.3 & 48.7 & 32.2 & \multirow[b]{2}{*}{$25.28 * * *$} \\
\hline No & 849 & 80.6 & 84.5 & 1.25 & 72.7 & 513 & 67.8 & \\
\hline \multicolumn{9}{|l|}{ Estudioscompleados } \\
\hline Primarios & 102 & 4.1 & & & 6.0 & 5.8 & 5.9 & \multirow{3}{*}{$8.64^{*}$} \\
\hline Secundarios & 36.7 & 40.8 & 37.1 & 5.20 & 33.3 & 23.7 & 31.2 & \\
\hline Universitarios & 52.7 & 54.1 & 52.9 & & 60.7 & 699 & 62.8 & \\
\hline \multirow{2}{*}{\multicolumn{9}{|c|}{ Con quien vive actualmente }} \\
\hline V ivo solo o sola & 102 & 14.3 & 10.6 & & & & & \multirow{3}{*}{$9.57 * *$} \\
\hline Con amigos of familia & 28.8 & 33.7 & 29.2 & 3.29 & 49.3 & 62.8 & 52.3 & \\
\hline Vivo en pareja & 61.0 & 52.0 & 60.2 & & 39.5 & 26.9 & 36.7 & \\
\hline \multicolumn{9}{|c|}{ Adividad laboral principal } \\
\hline A ma de casa & & 11.2 & 1.0 & \multirow{4}{*}{$114.22^{* * *}$} & .4 & 335 & 7.9 & \multirow{4}{*}{$185.84 * * *$} \\
\hline J ubilado/pensionista & 1.4 & 1.0 & 1.4 & & 9.0 & 2.6 & 7.6 & \\
\hline Estudiante & 5.9 & 6.1 & 5.9 & & 92 & 9.0 & 9.2 & \\
\hline Trabajador & 92.7 & 81.6 & 91.8 & & 81.4 & 54.8 & 75.4 & \\
\hline \multicolumn{9}{|c|}{ Hijos y/o dependientes a cargo } \\
\hline 0 & 472 & 54.1 & 47.8 & \multirow{4}{*}{7.88} & 32.6 & 43.6 & 35.1 & \multirow{4}{*}{$15.10^{* *}$} \\
\hline 1 & 213 & 11.2 & 20.4 & & 16.9 & 192 & 17.4 & \\
\hline 2 & 25.6 & 24.5 & 25.5 & & 19.1 & 212 & 19.6 & \\
\hline 3 o más & 6.0 & 10.2 & 6.3 & & 31.5 & 16.0 & 28.0 & \\
\hline \multicolumn{9}{|l|}{ Edad } \\
\hline 33 & 313 & 35.7 & 31.7 & \multirow{4}{*}{1.75} & 31.8 & 36.5 & 32.9 & \multirow{4}{*}{6.81} \\
\hline $34-39$ & 262 & 22.4 & & & & & & \\
\hline $40-46$ & 25.1 & 27.6 & 25.3 & & 19.3 & 21.8 & 19.9 & \\
\hline & 17.4 & 14.3 & 17.1 & & 30.5 & 19.9 & 28.1 & \\
\hline \multicolumn{9}{|l|}{$\frac{41}{\mathrm{IMC}}$} \\
\hline Bajopeso & 1.0 & 10.2 & 1.8 & \multirow{4}{*}{$58.27 * * *$} & 1.5 & 3.8 & 2.0 & \multirow{4}{*}{$36.18^{* * *}$} \\
\hline Pes o normal & 742 & 84.7 & 75.1 & & & 872 & & \\
\hline Sobrepeso & 239 & 5.1 & 22.3 & & $\begin{array}{l}30.6 \\
30.6\end{array}$ & 9.0 & 25.7 & \\
\hline Obesidad & .9 & & 8 & & 2.1 & & 1.6 & \\
\hline
\end{tabular}


Tabla 3. Medias, DT, F y p-valorde hansiedad cognitiva y variables bio-socio-demográficas, según sexo y país.

\begin{tabular}{|c|c|c|c|c|c|c|c|c|c|c|c|c|c|c|c|c|c|c|c|c|}
\hline & \multicolumn{10}{|c|}{ España } & \multicolumn{10}{|c|}{ México } \\
\hline & \multicolumn{5}{|c|}{$\begin{array}{l}\text { Hombres } \\
n=1007\end{array}$} & \multicolumn{5}{|c|}{$\begin{array}{c}\text { Mujeres } \\
n=98\end{array}$} & \multicolumn{5}{|c|}{$\begin{array}{c}\text { Hombres } \\
n=534\end{array}$} & \multicolumn{5}{|c|}{$\begin{array}{c}\text { Mujeres } \\
n=156\end{array}$} \\
\hline & $N$ & $M$ & $D T$ & $F$ & $p$ & $N$ & $M$ & $D T$ & $F$ & $p$ & $N$ & $M$ & $D T$ & $F$ & $p$ & $N$ & $M$ & $D T$ & $F$ & $p$ \\
\hline \multicolumn{21}{|l|}{ Tener entrenador } \\
\hline $\begin{array}{l}\text { Si } \\
\text { No }\end{array}$ & $\begin{array}{l}152 \\
855\end{array}$ & $\begin{array}{l}2.24 \\
1.95\end{array}$ & $\begin{array}{l}.68 \\
.66\end{array}$ & 24.38 & .000 & $\begin{array}{l}19 \\
79\end{array}$ & $\begin{array}{l}2.29 \\
2.03\end{array}$ & $\begin{array}{l}82 \\
.70\end{array}$ & 1.95 & .045 & $\begin{array}{l}146 \\
388\end{array}$ & $\begin{array}{l}2.04 \\
1.92\end{array}$ & $\begin{array}{l}.79 \\
.72\end{array}$ & 2.82 & .043 & $\begin{array}{l}76 \\
80\end{array}$ & $\begin{array}{l}2.07 \\
1.83\end{array}$ & $\begin{array}{l}.79 \\
.73\end{array}$ & 3.88 & .04 \\
\hline \multicolumn{21}{|l|}{ Estudioscompletados } \\
\hline Primarios & 103 & 2.03 & .70 & & & 4 & 2.50 & 1.16 & & & 32 & 2.25 & .91 & & & 9 & 2.06 & .97 & & \\
\hline ect & & 1.98 & .68 & .93 & .422 & 40 & 2.08 & .66 & 52 & .669 & 178 & 2.08 & .80 & 8.23 & .000 & 37 & 1.84 & .77 & .85 & .46 \\
\hline Universitarios & 531 & 1.99 & .66 & & & 53 & 2.05 & .75 & & & 324 & 1.85 & .67 & & & 109 & 1.98 & & & \\
\hline \multicolumn{21}{|l|}{ Con quien vive } \\
\hline Vivo solo o sola & 3 & 1.97 & .70 & & & 14 & 2.02 & .79 & & & 60 & 2.09 & .83 & & & 16 & 1.90 & .74 & & \\
\hline Con amigos of familia & 290 & 2.07 & .67 & 2.66 & .070 & 33 & 2.09 & .69 & .04 & .952 & 263 & 1.90 & .00 & 1.90 & .150 & $\begin{array}{l}10 \\
98\end{array}$ & $\begin{array}{l}1.90 \\
1.99\end{array}$ & 82 & .52 & .593 \\
\hline Vivo en pareja & 614 & 1.96 & .66 & & & 51 & 2.09 & .74 & & & 211 & 1.99 & .73 & & & 42 & 1.85 & .64 & & \\
\hline \multicolumn{21}{|l|}{ Acividadlaboral } \\
\hline Ama de casa & & & & \multirow{4}{*}{3.91} & \multirow{4}{*}{.020} & 11 & 2.56 & .78 & \multirow{4}{*}{3.26} & \multirow{4}{*}{.025} & & 2.20 & .00 & \multirow{4}{*}{.63} & \multirow{4}{*}{.591} & 52 & 2.03 & .79 & \multirow{4}{*}{.35} & \multirow{4}{*}{.785} \\
\hline J ubiladopensionista & 14 & 2. 12 & & & & 1 & 2.60 & & & & 48 & 1.86 & .73 & & & 4 & 1.95 & 1.10 & & \\
\hline Estudiante & 59 & 2.22 & .74 & & & 6 & 2.53 & 1.01 & & & 49 & 206 & 67 & & & 14 & 191 & 1.02 & & \\
\hline Trabajador & 933 & 1.97 & .66 & & & 80 & 1.98 & .66 & & & 433 & 1.95 & .76 & & & 85 & $\begin{array}{l}1.31 \\
1.89\end{array}$ & 1.02 & & \\
\hline \multicolumn{21}{|l|}{$\mathrm{Hijos}$, dependientes } \\
\hline Milos, & 475 & 1.99 & .67 & \multirow{4}{*}{.84} & \multirow{4}{*}{.472} & 53 & 2.19 & .71 & \multirow{4}{*}{91} & & 174 & 1.97 & .74 & & & 68 & 2.07 & .82 & & \\
\hline & 214 & 2. 01 & .68 & & & 11 & 1.89 & 83 & & .435 & 90 & 1.95 & .80 & .34 & .794 & 30 & 1.99 & .77 & 1.56 & .20 \\
\hline & & 1.95 & .65 & & & 24 & 1.97 & .76 & & .435 & 102 & 2.00 & .72 & .34 & .794 & 33 & 1.80 & .68 & & \\
\hline 3 o más & & 2.09 & .77 & & & 10 & 1.98 & .60 & & & 168 & 1.91 & .73 & & & 25 & 1.75 & & & \\
\hline Edad & & & & & & & & & & & & & & & & & & & & \\
\hline$<3$ & & 2.58 & 1.08 & & & 35 & 2.38 & .74 & & & 170 & 1.98 & .73 & & & 57 & 2.16 & .83 & & \\
\hline $34-39$ a & & 2.48 & 1.17 & & & 22 & 1.81 & 46 & & & 98 & 1.95 & .71 & & 056 & 34 & 1.77 & .76 & & 040 \\
\hline 40-46 años & 253 & 2.30 & 1.09 & 7.24 & .000 & 27 & 1.89 & .69 & 3.94 & .011 & 103 & 1.93 & .75 & .10 & .956 & 34 & 1.78 & .62 & 2.66 & .04 \\
\hline$>47$ años & 175 & 2.24 & 121 & & & 14 & 2.11 & 86 & & & 163 & 1.94 & .78 & & & ${ }_{31}^{34}$ & 1.94 & .02 & & \\
\hline 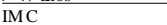 & & & & & & & & & & & & & & & & & & & & \\
\hline $\mathrm{Ba}$ & . & 2.22 & & & & 10 & 2.22 & .86 & & & 8 & 2.42 & .58 & & & 6 & 1.83 & .87 & & \\
\hline Pes o normal & 747 & 2.02 & .68 & & & 83 & 2.08 & .72 & & 590 & 351 & 1.97 & .77 & & & 136 & 1.97 & .78 & & \\
\hline & 241 & 1.89 & .64 & 4.56 & .004 & 5 & 1.80 & .64 & 54 & .580 & 163 & 1.92 & .68 & 1.45 & .225 & 14 & 1.72 & .56 & 73 & 48 \\
\hline Obes & 9 & 1.48 & .40 & & & & & & & & 0 & 1.76 & 75 & & & & & & & \\
\hline
\end{tabular}

Tabla 4. Medias, $D T$, F y p-valor de la ansiedad somática y variables bio-socio-demográficas, según sexo y país.

\begin{tabular}{|c|c|c|c|c|c|c|c|c|c|c|c|c|c|c|c|c|c|c|c|c|}
\hline \multirow[b]{4}{*}{ ener entrenador } & \multicolumn{10}{|c|}{ España } & \multicolumn{10}{|c|}{ México } \\
\hline & \multicolumn{5}{|c|}{$\begin{array}{l}\text { Hombres } \\
n=1007\end{array}$} & \multicolumn{5}{|c|}{$\begin{array}{c}\text { Mujeres } \\
n=98\end{array}$} & \multicolumn{5}{|c|}{$\begin{array}{c}\text { Hombres } \\
n=534\end{array}$} & \multicolumn{5}{|c|}{$\begin{array}{c}\text { Mujeres } \\
n=156\end{array}$} \\
\hline & $N$ & $M$ & $D T$ & $F$ & $p$ & $N$ & $M$ & $D T$ & $F$ & $p$ & $N$ & $M$ & $D T$ & $F$ & $p$ & $N$ & $M$ & $D T$ & $F$ & $p$ \\
\hline & & & & & & & & & & & & & & & & & & & & \\
\hline $\begin{array}{l}\mathrm{Si} \\
\mathrm{No}\end{array}$ & $\begin{array}{l}152 \\
855\end{array}$ & $\begin{array}{l}2.39 \\
2.22\end{array}$ & $\begin{array}{l}.71 \\
72\end{array}$ & 6.95 & .008 & $\begin{array}{l}19 \\
79\end{array}$ & $\begin{array}{l}2.67 \\
2.29\end{array}$ & .69 & 4.40 & .038 & $\begin{array}{l}146 \\
388\end{array}$ & $\begin{array}{l}2.29 \\
2.14\end{array}$ & .78 & 4.10 & .043 & $\begin{array}{l}76 \\
80\end{array}$ & $\begin{array}{l}2.21 \\
2.17\end{array}$ & .74 & .08 & .766 \\
\hline Estudios completa & & & & & & & & & & & & & & & & & & & & \\
\hline Primarios & 103 & 2.35 & .73 & & & 4 & 3.04 & .70 & & & 32 & 2.34 & .82 & & & 9 & 2.27 & .81 & & \\
\hline cunc & 370 & 2.21 & .75 & 1.10 & .347 & 40 & 2.43 & .71 & 1.66 & .180 & 178 & 2.28 & .82 & 3.57 & .029 & 37 & 2.10 & .79 & 1.05 & 369 \\
\hline Unive & 531 & 2.25 & .70 & & & 53 & 2.26 & .73 & & & 324 & 2.11 & .75 & & & 109 & $\begin{array}{l}2.10 \\
2.22\end{array}$ & .76 & & .509 \\
\hline Con qu & & & & & & & & & & & & & & & & & & & & \\
\hline Vivo s & 103 & 2.18 & .78 & & & 14 & 2.03 & .62 & & & 60 & 2.33 & .76 & & & 16 & 2.02 & & & \\
\hline Con am & 290 & 2.27 & .74 & .54 & .582 & 33 & 2.47 & .69 & 1.84 & .163 & 263 & 2.12 & .77 & 2.13 & .119 & 98 & 2.17 & .80 & .86 & .423 \\
\hline Vivo en pare & 614 & 2.24 & .70 & & & 51 & 2.38 & .76 & & & 211 & 2.21 & .79 & & & 42 & 2.30 & .80 & & \\
\hline$\overline{\text { Adivil }}$ & & & & & & & & & & & & & & & & & & & & \\
\hline ma & 14 & 2.29 & & & & & 2.53 & .92 & & & 2 & 2.58 & 58 & & & & & & & \\
\hline Jubilado/pensi onista & 59 & 2.45 & .75 & & & 1 & 2.33 & & & & 48 & 2.02 & .81 & & & 4 & 2.66 & 1.15 & & \\
\hline Estudiante & 933 & 2.23 & .72 & 2.68 & .069 & 6 & 2.66 & .84 & .62 & .602 & 49 & 2.27 & .74 & 1.01 & .385 & 14 & 2.08 & .99 & 1.41 & .240 \\
\hline Traba & & & & & & 80 & 2.32 & .69 & & & 433 & 2.18 & .78 & & & 85 & 2.11 & .70 & & \\
\hline endientes & & & & & & & & & & & & & & & & & & & & \\
\hline & 475 & 2.22 & .72 & & & 53 & 2.43 & & & & 174 & & & & & 68 & 22 & & & \\
\hline 1 & 214 & 2.20 & .70 & 135 & 255 & 11 & 2.33 & 1.01 & 11 & 348 & 90 & 2.07 & .78 & 74 & 528 & 30 & 2.22 & .84 & 18 & 908 \\
\hline & 258 & 2.30 & .74 & & & 24 & 2.14 & .62 & & .348 & 102 & 2.21 & .77 & & & 33 & 2.10 & .77 & & .908 \\
\hline 3 o más & 60 & 2.34 & .69 & & & 10 & 2.55 & .60 & & & 168 & 2.20 & .81 & & & 25 & 2.19 & .73 & & \\
\hline $\begin{array}{l}\text { Edad } \\
<33 \text { anos }\end{array}$ & 315 & 2.34 & .73 & & & 35 & 2.57 & 69 & & & 170 & 2.24 & 74 & & & 57 & 226 & 77 & & \\
\hline 34-3s & & 2.27 & .71 & 428 & 005 & 22 & 2.12 & .80 & 225 & 047 & 98 & 2.17 & .78 & 71 & 542 & 34 & 2.12 & .72 & 28 & .839 \\
\hline & & 2.19 & .66 & & & 27 & 2.23 & .52 & & & 103 & 2.17 & .76 & & & 34 & 2.13 & .75 & & \\
\hline$>47$ añ & 175 & 2.11 & .77 & & & 14 & & 01 & & & 163 & 2.12 & .83 & & & 31 & 2.20 & .87 & & \\
\hline & & & & & & & & & & & & & & & & & & & & \\
\hline & 10 & 2.16 & .71 & & & 10 & 2.31 & 1.02 & & & 0 & 2.83 & .78 & & & 6 & 2.13 & .67 & & \\
\hline Pesono & 747 & 2.26 & .72 & 48 & & 83 & 2.34 & .69 & & & 351 & 2.17 & .75 & & .052 & 136 & 2.21 & .79 & 69 & . 502 \\
\hline & 241 & 2.20 & .72 & .48 & .691 & 5 & 2.86 & .59 & 1.24 & .292 & 163 & 2.13 & .81 & 2.59 & .052 & 14 & 1.96 & .69 & & \\
\hline bes idad & 9 & 2.16 & .91 & & & & & & & & 11 & 2.48 & 1.07 & & & & & & & \\
\hline
\end{tabular}

Tabla 5. Medias, $D T, F$ y $p$-valor de la autoconfianza y variables bio-socio-demográficas, según sexo y país.

\begin{tabular}{|c|c|c|c|c|c|c|c|c|c|c|c|c|c|c|c|c|c|c|c|c|}
\hline \multirow{3}{*}{ Tener entrepador } & \multicolumn{10}{|c|}{ España } & \multicolumn{10}{|c|}{ México } \\
\hline & \multicolumn{5}{|c|}{$\begin{array}{c}\text { Hombres } \\
n=1007\end{array}$} & \multicolumn{5}{|c|}{$\begin{array}{c}\text { Mujeres } \\
n=98\end{array}$} & \multicolumn{5}{|c|}{$\begin{array}{c}\text { Hombres } \\
n=534\end{array}$} & \multicolumn{5}{|c|}{$\begin{array}{l}\text { Mujeres } \\
n=156\end{array}$} \\
\hline & $N$ & $M$ & $D T$ & $F$ & $p$ & $N$ & $M$ & $D T$ & $F$ & $p$ & $N$ & $M$ & $D T$ & $F$ & $p$ & $N$ & $M$ & DT & $F$ & $p$ \\
\hline & & & & & & & & & & & & & & & & & & & & \\
\hline & $\begin{array}{l}152 \\
855\end{array}$ & $\begin{array}{l}3.24 \\
3.12\end{array}$ & $\begin{array}{l}.58 \\
.57\end{array}$ & 5.2 & .022 & $\begin{array}{l}19 \\
79\end{array}$ & $\begin{array}{l}2.86 \\
3.01\end{array}$ & $\begin{array}{l}.92 \\
.65\end{array}$ & .71 & .400 & $\begin{array}{l}146 \\
388\end{array}$ & $\begin{array}{l}3.61 \\
3.54\end{array}$ & $\begin{array}{l}.51 \\
.58\end{array}$ & 1.35 & .046 & $\begin{array}{l}76 \\
80\end{array}$ & $\begin{array}{l}3.46 \\
3.45\end{array}$ & $\begin{array}{l}.61 \\
.66\end{array}$ & .01 & 918 \\
\hline \multicolumn{21}{|c|}{ Es tudios completados } \\
\hline Pivame & & 3.25 & .63 & & & 4 & 2.95 & 1.21 & & & 32 & 3.60 & .47 & & & 9 & 3.46 & .68 & & \\
\hline and & 0 & 3.18 & .57 & 2.75 & .042 & 40 & 3.05 & .75 & 27 & .846 & 178 & 3.57 & .58 & .09 & .911 & 37 & 3.47 & .71 & .26 & 854 \\
\hline Universitarios & 531 & 3.10 & .56 & & & 53 & 2.94 & .65 & & & 324 & 3.55 & 5.57 & & & 109 & 3.44 & .60 & & \\
\hline \multicolumn{21}{|l|}{ Con quien vi } \\
\hline Vivo solo o & & 3.10 & .60 & & & 14 & 2.90 & .67 & & & 60 & 3.58 & 3.59 & & & 16 & 3.60 & .64 & & \\
\hline Con am & 290 & 3.16 & .59 & 39 & .674 & 33 & 2.96 & .67 & 20 & .819 & 263 & 3.58 & .55 & .23 & .788 & 98 & 3.58 & .44 & 10.02 & .000 \\
\hline Vivoe & 614 & 3.14 & .57 & & & 51 & 3.02 & .75 & & & 211 & 3.54 & .58 & & & 42 & 3.10 & .85 & & \\
\hline \multicolumn{21}{|l|}{ Actividadlaboral } \\
\hline na de casa & & & & \multirow{4}{*}{5} & \multirow{4}{*}{.469} & 11 & 3.10 & .76 & \multirow{4}{*}{1.38} & \multirow{4}{*}{.252} & & 3.40 & .84 & \multirow{4}{*}{.12} & \multirow{4}{*}{.945} & 52 & 3.48 & .63 & \multirow{4}{*}{33} & \multirow{4}{*}{.477} \\
\hline ta & 14 & 3.32 & .30 & & & 1 & 3.20 & & & & 48 & 3.60 & .51 & & & 4 & 3.00 & 1.01 & & \\
\hline Estudiante & 59 & 3.11 & .69 & & & & 2.43 & .81 & & & 49 & 3.55 & .54 & & & 14 & 3.37 & .84 & & \\
\hline abaj: & 933 & 3.14 & .57 & & & 80 & 3.01 & .69 & & & 433 & 3.56 & & & & 85 & 3.47 & .57 & & \\
\hline \multicolumn{21}{|l|}{ Hijos dependientes } \\
\hline & 475 & 3.16 & .57 & \multirow{4}{*}{7} & \multirow{4}{*}{.846} & 53 & 2.95 & .75 & \multirow{4}{*}{30} & & 174 & 3.57 & .55 & & & 68 & 3.43 & .58 & & \\
\hline & 214 & 3.12 & .55 & & & 11 & 3.07 & .88 & & & 90 & 3.53 & .60 & .19 & & 30 & 3.58 & .60 & 2.38 & .071 \\
\hline & 258 & 3.13 & .59 & & & 24 & 2.94 & .61 & & .824 & 102 & 3.55 & .60 & .19 & .897 & 33 & 3.58 & 42 & & \\
\hline 30 & 60 & 3.15 & .65 & & & 10 & 3.16 & .58 & & & 168 & 3.58 & .53 & & & 25 & 3.19 & & & \\
\hline & & & & & & & & & & & & & & & & & & & & \\
\hline$<33 \mathrm{a}$ & & 3.18 & & & & 35 & 2.92 & .77 & & & 170 & 3.58 & .55 & & & 57 & 3.43 & .58 & & \\
\hline $34-39$ & 264 & 3.16 & .55 & & & 22 & 2.80 & .67 & & 278 & 98 & 3.60 & .59 & 61 & 607 & 34 & 3.50 & .65 & .13 & 939 \\
\hline $\begin{array}{l}40-46 \text { años } \\
>47 \text { años }\end{array}$ & 253 & 3.13 & .58 & 1.70 & .164 & 27 & 3.15 & .61 & 1.30 & & 103 & 3.57 & .51 & & & 34 & 3.47 & .73 & & \\
\hline $47,-3$ & 175 & 3 & .5 & & & 14 & 3.12 & 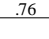 & & & 163 & 1 & L & & & 31 & 3.41 & .62 & & \\
\hline & & 3.20 & .63 & & & 10 & 2.66 & .81 & & & 8 & 3.77 & .63 & & & 6 & 3.63 & .29 & & \\
\hline so normal & 747 & 3.15 & .57 & & & 83 & 3.05 & .68 & & & 351 & 3.59 & .56 & & & 136 & 3.46 & .61 & & \\
\hline & 241 & 3.10 & .59 & .71 & .544 & 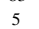 & 2.60 & .86 & 2.16 & .120 & 163 & 3.48 & 3.59 & 1.98 & .116 & 14 & 3.32 & .93 & .51 & 599 \\
\hline & $\begin{array}{c}411 \\
9\end{array}$ & $\begin{array}{r}0.10 \\
\end{array}$ & .48 & & & & & & & & 11 & 3.63 & . & & & & & & & \\
\hline
\end{tabular}

En ansiedad somática se obtuvieron valores medios en todas las opciones de respuesta de las variables analizadas, tanto en la muestra española como en la mexicana. En la muestra española, además, se obtuvieron algunas diferencias significativas, de forma que esta ansiedad somática disminuía en hombres y mujeres sin entrenador y mayores de 47 años (hombres) o de entre 34 y 39 años (mujeres). En la muestra mexicana, sin embargo, sólo hubo diferencias significativas en aquellos hombres que no tenían entrenador y tenían un mayor nivel de estudios, en los cuales su ansiedad somática disminuía(Véase Tabla 4).

En autoconfianza los valores obtenidos son altos en la muestra española y muy altos en la muestra mexicana, en ambos casos con diferencias significativas a favor de los varones. En la muestra española, además, se obtuvieron diferencias significativas en aquellos corredores varones con entrenador y menor nivel de estudios, en los cuales aumentaba su autoconfianza, mientras que en la muestra mexicana lo hacía únicamente en los varones con entrenador y en las mujeres que vivían solas (Véase Tabla 5).

\section{Discusión y conclusiones}

A pesar de ser un deporte individual (Furst y Tenembaum, 1984), los moderados valores de ansiedad precompetitiva hallados en la población de corredores de ruta española y mexicana, podrían quedar explicados por el hecho de que la mayoría de los corredores, al no ser de élite, simplemente tengan como objetivo el terminar la prueba, preferiblemente en un tiempo mejor que la última vez que finalizaron una.

De la misma manera, como explicaban Arbinaga y Caracuel (2005), al tratarse en la mayoría de los casos de deportistas adultos y mayores, que además tienen la tranquilidad de tener trabajo (Tabla 2), hace que su ansiedad disminuya y aumente su autoconfianza. Indudablemente, el hecho de quela inmensa mayoría de la muestra sean varones, como explicaban Ponce de León et al. (2006), puede haber influido significativamente en la moderada ansiedad mostrada.

Otro factor que puede haber influido en las moderadas puntuaciones de la ansiedad es el ambiente en el que se desarrolla la carrera, como explicaban Arbinaga y Caracuel (2005), puesto que, lejos de la presión del público de los estadios propia de los deportes de equipo, la competición se desarrolla recorriendo los lugares más bellos de la ciudad con poca presencia de público a ambos lados del recorrido, que además van dejando atrás según avanzan.

También, como explicaba Jaenes (2000), las características de la prueba, donde la mayoría de los corredores corren en grupos de amigos animándose mutuamentepara finalizar, haceque la afronten con menor ansiedad y mayor autoconfianza.

Igualmente, el hecho de que la inmensa mayoría de los corredores no se preocupe de ser asesorado por un entrenador (Tabla 2), evi- 
dencia nuevamente que la mayoría de ellos no busquen un alto rendimiento en la prueba, sino simplemente finalizarla, lo cual puede favorecer el aumento de la autoconfianza y la disminución de la ansiedad.

A modo de conclusiones, puede afirmarse que los corredores de ruta tienen una moderada ansiedad precompetitiva, tanto cognitiva como somática, sin diferencias significativas entre sexos ni entre las muestras española y mexicana. Sin embargo, los valores obtenidos en autoconfianza son altos en la muestra española y muy altos en la muestra mexicana, en ambos casos con diferencias significativas a favor de los varones. Además, la influencia que tienen las variables bio-socio-demográficas en las puntuaciones de la ansiedad cognitiva, ansiedad somática y autoconfianza, existen numerosas diferencias significativas, tanto por sexo como entre las muestras española y mexicana.

Por todo ello, se hace necesario seguir investigando a esta creciente población en futuras líneas de investigación que incluyan entre las variables influyentes o predictoras de la ansiedad precompetitiva a diversos constructos psicológicos (motivaciones, niveles de satisfacción intrínseca, adicción al entrenamiento, percepción y/o creencias sobre las causas del éxito deportivo, etc.) o variables de entrenamiento (número de días que entrena por semana, número de horas que entrena por sesión, número de compañeros de entrenamiento, mejor marca personal en medio maratón y/ maratón, etc.).

\section{Referencias}

Anshel, M., Freedson, P., Hamill, J., Haywood, K., Horvat, M., \& Plowman, S. (1991). Dictionary of the sport and exercise sciences. Champaign, IL: Human Kinetics.

Arbinaga, F, \& Caracuel, J. C. (2005). Precompetición y ansiedad en fisicoculturistas. Revista de Psicología del Deporte, 14(2), 195-208.

Buceta, J., López, A., Pérez-Llantada, M., Vallejo, M., \& Del Pino, M. (2003). Estado psicológico de los corredores populares de maratón en los días anteriores a la prueba. Psicothema, 15, 273-277.

Furst, D. M., \& Tenembaum, G. (1984). Sport performance and various state anxiety components: Across sectional study. Netanya: Wingate Institute.

Gutiérrez, M., Estévez, A., García, J., \& Pérez, H. (1997). Ansiedad y rendimiento atlético en condiciones de estrés: efectos moduladores de la práctica. Revista de Psicología del Deporte, 6(2), 27-46.

Jaenes, J. C. (2000). Estado emocional y conducta deportiva: Ansiedad competitiva en corredores de maratón. Tesis Doctoral. Universidad deSevilla.
Jones, J. G. (1991). Recent developments and current issues in competitive state anxiety research. The Sport Psychologist, 4, 152155.

Jones, G, \& Swain, A. (1995). Predisposition to experience debilitative and facilitative anxiety in elite and non-elite performers. The Sport Psychologist, 9, 201-211.

Martens, R., Burton, D., Vealey, R., Bump, L., \& Smith, D. (1990). Development and validation of the Competitive State Anxiety Inventory-2. En R. Martens, R. S. Vealey \& D. Burton (Eds.): Competitive anxiety in sport (pp. 117-190). Champaign, IL: Human Kinetics.

Mellalieu, S., Hanton, S., \& O’Brien, M. (2004). Intensity and direction of competitive anxiety as a function of sport type and experience. Scandinavian Journal of Medicine and Science in Sports, 14, 326334.

Ponce de León, Y., López, J., \& Medina, M. (2006). Habilidades psicológicas en los atletas de primera fuerza en atletismo. Revista de Ciencias del Ejercicio-FOD-, 2, $42-57$.

Pozo, A. (2007). Intensidad y dirección de la ansiedad competitiva y expectativas de resultados en atletas y nadadores. Revista de Psicología del deporte, 16(2), 137-150.

Real Federación Española deAtletismo (2013). Calendario Nacional de Competiciones. Disponible en http://www.rfea.es/web/competiciones/competiciones.asp?mes=-1

Scanlan, T. K., Babkes, M. L., \& Scanlan, L.A. (2005). Participation in sport: A developmental glimpse at emotion. En J. L. Mahoney, R. W. Larson \& J. S. Eccles (Eds.). Organized activities as contexts of development: Extracurricular activities, after school, and community programs. (pp. 275-309). Mahwah, NJ: Erlbaum.

Scanlan, T. K., \& Lewthwaite, R. (1986). Social psychological aspects of competition for male youth sport participants: IV. Predictors of enjoyment. Journal of Sport Psychology, 8, 25-35.

Smith, R. (1989). Athletic stress and burnout: conceptual models and intervention strategies. En D. Hackfort \& C. D. Spielberger (eds.): Anxiety in sports: An international perspective (pp. 183-201). Nueva York, NY: Hemisphere.

Smith, R. E., Smoll, F. L., \& Wiechman, S. (1998). Measurement of trait anxiety in sport. En J. L. Duda (Ed.), Advances in sport and exercise psychology measurement. Morgantown, WV: Fitness Information Technology.

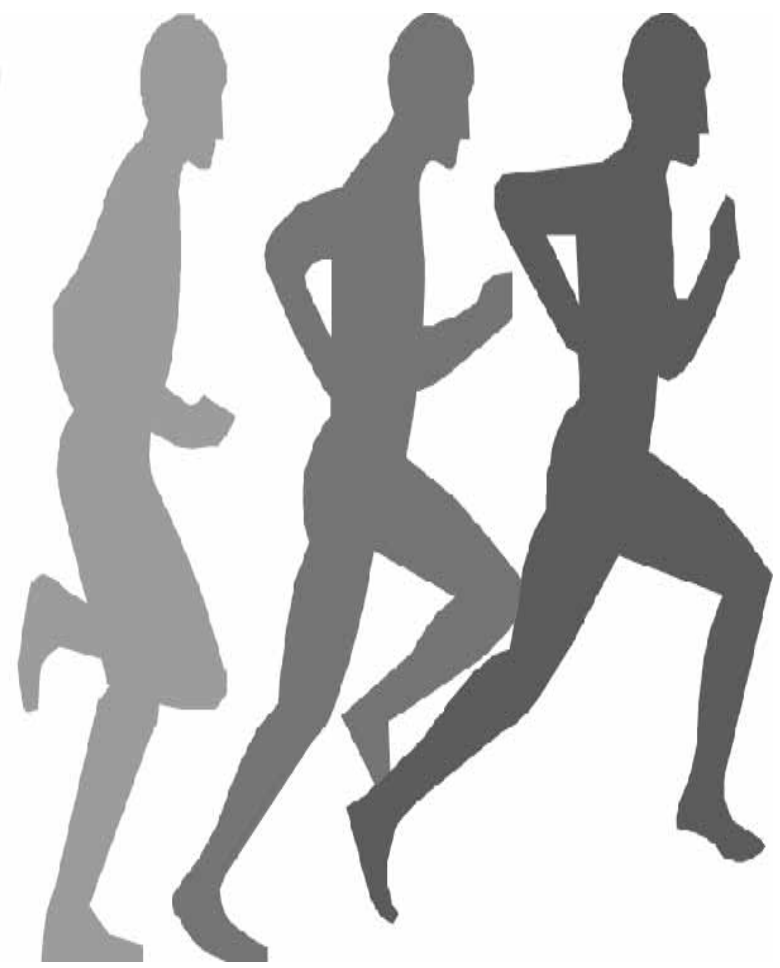

